* Chefe do Departamento de Produção e de Operaçốes Industriais (POI) da Escola de Administração de Empresas de São Paulo da Fundação Getulio Vargas.
Numa empresa, itens múltiplos são definidos como itens do estoque, que são empregados em múltiplos de um item unitário - por exemplo, um carrinho de quatro rodas necessita de uma caçamba, dois eixos, quatro rodas $e$, eventualmente, uma melhoria do projeto do produto, mancais e rolamentos em número também de quatro, ou mesmo rodas de borracha. Ora, uma das críticas principais de Burbidge ${ }^{1}$ ao sistema de administração de estoques por meio do lote econômico, tanto de produção quanto de compras é a impos- . sibilidade de conseguir, pela sistemática adotada, múltiplos necessários à boa ordem de produção, para que náo haja nem falta, nem excesso de itens, que formarâo um conjunto em proporçôes definidas. $^{2}$

Realmente, uma parada da linha de montagem de determinado conjunto pode deixar grupos de peças obsoletas, que não se prestam a outra utilidade, nem podem ser aproveitados para uma receita eventual como peças sobressalentes dos conjuntos originais vendidos, por ter uma vida longa. $\mathrm{Na}$ imensa literatura sobre $\mathrm{O}$ assunto $\mathrm{O}$ autor não conseguiu encontrar até hoje nenhuma referència a um método para estabelecer um lote que minimiza o custo anual total das compras para o conjunto montado, lote este diferente do lote econômico de compras, mas resultando numa soma de custos mínimos para todos os itens que entram no conjunto.

O autor cedo verificou que não é viável o estabelecimento de um lote múltiplo de pecas fabricadas, pois o lote económico de fabricaçäo depende demasiadamente do custo de preparaçáo. Quanto a este, não é possível estabelecer nem uniformidade entre diversos itens nem manter esta uniformidade durante determinado espaço de tempo. Realmente, - custo de preparação numa linha de produção, que trabalha com tempo ocioso, é exclusivamente o da mão-de-obra de planejamento e de preparaçăo da máquina, e da desmontagem eventual dos dispositivos depois do uso. No entanto, com uma linha em pleno uso de sua capacidade, deve ser considerado como custo de preparaçăo, uma espécie de lucro cessante da operação nela realizada, ou da média de operações realizadas. ${ }^{3}$ A variabilidade assim descrita impossibilita a criação de uma fórmula de lote económico de produção, mas não fórmulas para o lote de compras em múltiplos.

Suponhamos que numa empresa haja necessidade de compras anuais para uso uniforme de material parà a construção de 3.600 carrinhos de quatro rodas. As peças necessárias para a montagem são para cada caçamba dois eixos e quatro rodas. Considerando um custo de comprar $S$ de $C r \$ 450,00$ e uma taxa de manutençäo de estoque $I=0,36$ (36\%) pode ser estabelecido o seguinte quadro do lote econômico por item. ${ }^{4}$ 


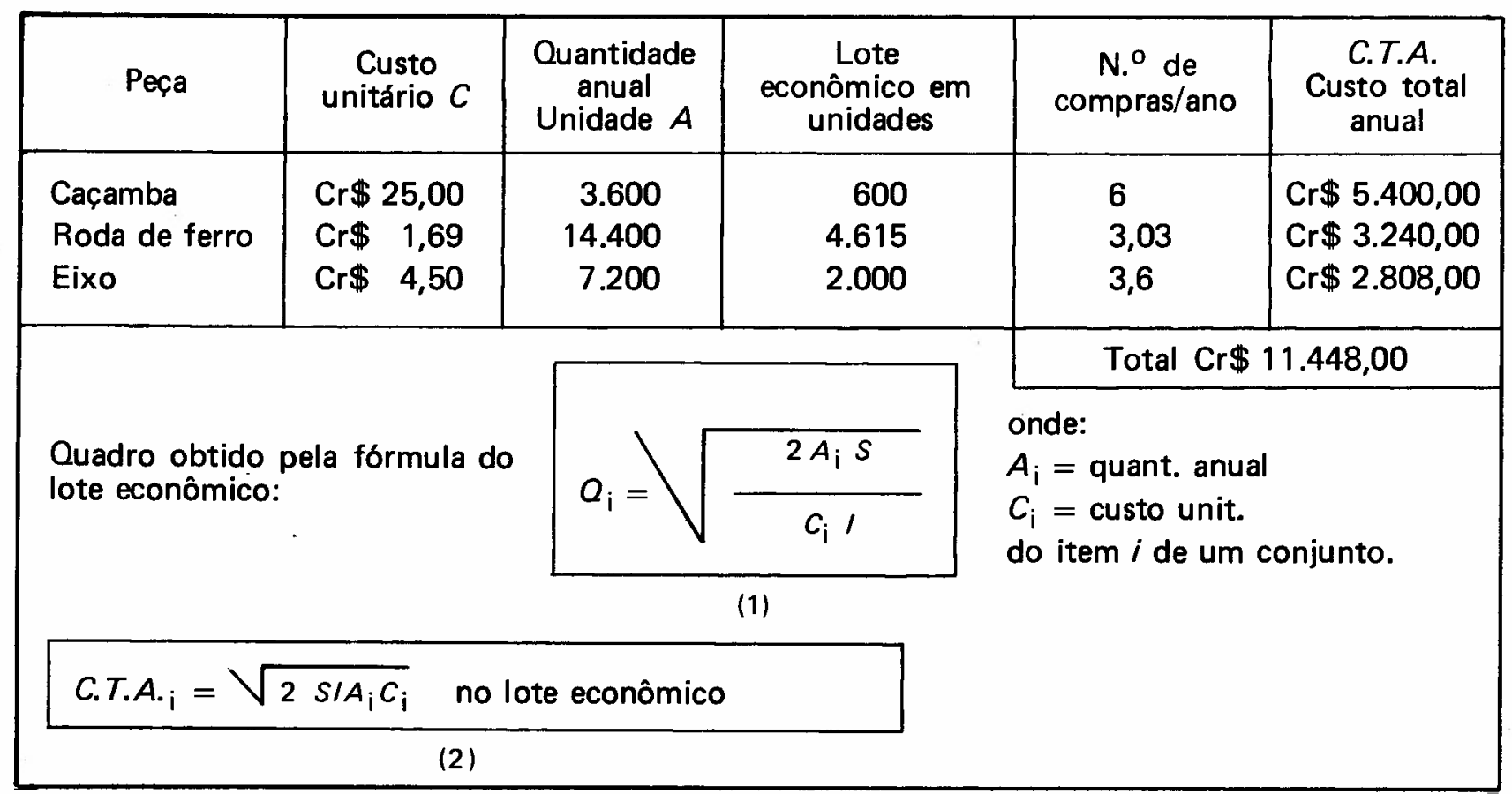

Uma das tendências mais comuns para o estabelecimento de lote múltiplo é tomar o item mais caro, e a partir dele estabelecer múltiplos. ${ }^{5}$ Portanto, ter-se-ia a partir do quadro acima para 600 caçambas a compra de $\mathbf{2 . 4 0 0}$ rodas e 1.200 eixos, seis vezes por ano, com um aumento do custo de manter estoque e comprar. Infelizmente, essa solução não é a melhor como será demonstrado no exemplo.

Parece simples o estabelecimento de uma fórmula geral que dá um custo total anual mínimo, não tendo, eventualmente, nenhum lote individual econômico na sua composição. Um conjunto composto de $i$ itens, que são numerados de 1 a $i$, terá sua fórmula derivada da mesma maneira como a do lote econômico, por meio de uma derivação relativa a uma quantidade e igualando o resultado da derivação a zero, portanto, fazemos nada mais do que o método clássico de encontrar o mínimo de uma função contínua. Ora, a soma de $i$ funçőes contínuas (de custo anual) ainda é uma função contínua, e, assim, o método pode ser aplicado.

O importante na dedução desta fórmula é fixar arbitrariamente, como base de cálculo de minimização do custo, o item de menor quantidade anual. Numeramos este item $A_{1}$ numa série que vai de $A_{1}, A_{2}, A_{3}, \ldots$ até $A_{\mathrm{j}}$. Como qualquer consumo anual é um múltiplo de $A_{1}$ necessário para a montagem de conjuntos, temos então:

$$
\begin{aligned}
& A_{1}=f_{1} \cdot A_{1} \\
& A_{2}=f_{2} \cdot A_{1} \\
& A_{3}=f_{3} \cdot A_{1} \\
& \ddot{A}_{i}=f_{i} \cdot A_{1}
\end{aligned}
$$

onde $f_{1}, f_{2}, f_{3} \ldots f_{\mathrm{i}}$ são fatores múltiplos, usualmente números inteiros e onde por conveniência se coloca $f_{1}=1$. $O$ múltiplo não será inteiro quando temos por exemplo 1,5 litros de tinta por caçamba e a quantidade anual de tinta ficaria em 5.400 litros. Por sua vez, a quantidade de compra cada vez fica:

$$
\begin{aligned}
& Q_{1}=f_{1} Q_{1} \\
& Q_{2}=f_{2} Q_{1} \\
& \ldots \ldots \ldots \ldots . . . \\
& \ddot{Q_{\mathrm{i}}}=f_{\mathrm{i}} \ddot{Q}_{1}
\end{aligned}
$$

Fora do lote econômico o custo total anual de comprar fica com o primeiro termo lo custo de $N$ compras) e o segundo termo, o custo de estocar.

$$
\text { C.T.A. } \mathrm{i}=\frac{A_{\mathrm{i}}}{f_{\mathrm{i}} \times Q_{1}} \times S+\frac{f_{\mathrm{i}} Q_{1} C_{\mathrm{i}} l}{2}
$$

onde

$$
\frac{A_{\mathrm{i}}}{f_{\mathrm{i}} \times Q_{1}}=N
$$

o custo total anual de todos os itens do conjunto é o somatório:

$$
{\underset{1}{\Sigma}}_{i}^{i} \text { C.T.A. } \mathrm{i}=\frac{S}{Q_{1}} \sum_{1}^{i}\left(\frac{A_{\mathrm{i}}}{f_{\mathrm{i}}}\right)+\frac{1 Q_{1}}{2} \sum_{1}^{i} f_{i} C_{i}
$$

o mínimo de $\sum_{1}^{\mathrm{i}}$ C.T.A. $\mathrm{i}$ se obtém derivando em em relação a $Q_{1}$ e igual a zero: 
$d(\stackrel{i}{\Sigma}$ C.T.A. $i)$

$\frac{1}{d Q_{1}}=-\frac{1}{Q_{1}^{2}} \sum_{1}^{j}\left(\frac{A_{i}}{f_{i}}\right)+\frac{1}{2} \Sigma f_{i} C_{i}$

ora: $\frac{A_{i}}{f_{\mathrm{i}}}=A_{1}$, pois $A_{i}=f_{1} A_{1}$

então: $\frac{S}{Q_{1}^{2}} i A_{1}=\frac{l}{2} \sum_{1}^{i} f_{i} C_{\mathrm{i}}$

esclarecendo que $\sum_{1}^{i}\left(\frac{A_{i}}{f_{i}}\right)=\frac{A_{1}}{f_{1}}+\frac{A_{2}}{f_{2}}+\cdots+\frac{A_{i}}{f_{i}}$

ou seja $i$ vezes $A_{1}$

então: $Q_{1}^{2}=\frac{2 S}{l} \times \frac{i \times A_{1}}{\sum_{1}^{i} f_{i} C_{i}}$

ou $a_{1}=\sqrt{\frac{2 S}{I}} \cdot \sqrt{\frac{i A_{1}}{\sum_{1}^{i} f_{i} C_{i}}}$

Exemplos:

$$
\text { Caso } \mathrm{i}=\mathbf{2} \text { para "caçamba e rodas" temos }
$$

$$
\begin{array}{r}
f_{i}=1 \quad a_{1}=\sqrt{\frac{2 S}{I}} \cdot \sqrt{\frac{i A_{1}}{\frac{i}{1} f_{j} C_{i}}} \\
a_{1}=\sqrt{\frac{2 \times 450}{0,36}} \cdot \sqrt{\frac{2 \times 3600}{25+4 \times 1,69}} \cong 750 \\
\frac{3600}{750}=4,8 \text { compras/ano }
\end{array}
$$

donde: $Q_{2}=f_{2} \times Q_{1}=4 \times 750=3.000$ unidades

$$
\begin{aligned}
& \text { Caso } i=3 \\
& Q_{1}=\sqrt{\frac{2 \times 450}{0,36}} \cdot \sqrt{\frac{3 \times 3.600}{25+4 \times 1,69 \times 4,5}} \cong 814 \\
& \text { unidades }
\end{aligned}
$$

$Q_{1}=814$ unidades

$Q_{2}=2 \times 814=1.628$ unidades

$Q_{3}=4 \times 814=3.256$ unidades $\frac{3.600}{814}=4,42$

compras/ano
O custo total dessas 4,42 compras será $4,42 \times 450 \times 3$ para os três itens enquanto o custo de ter o estoque será respectivamente:

$\frac{814 \times 25 \times 0,36}{2}, \frac{1.628 \times 4,5 \times 0,36}{2} \mathrm{e}$

$\underline{3.256 \times 1,69 \times 0,36}$

2

Então o C.T.A. será:

$4,42 \times 450 \times 3+\frac{814 \times 0,36}{2}(25+2 \times 4,5+4 \times 1,69)=$ $=5.967+146,52 \times(25+9+6,76)=5.867+$ $+146,52 \times 40,76=5.967+5.972=11.948,15$

Conseqüentemente houve um encarecimento de $\mathrm{Cr} \$ 11.948-11.446=\mathrm{Cr} \$ 502$ ou $4,4 \%$

4,4\% de encarecimento não são demasiados considerando as vantagens possíveis pela compra em unidades múltiplas.

A alternativa de tomar o item mais caro e comprar múltiplos dele ficava em:

- caçamba:

$\operatorname{Cr} \$ 5.400,00$

- roda de

ferro:

$6 \times 450+\frac{2.400 \times 0,36 \times 1,69}{2}=\operatorname{Cr} \$ 3.329,00$

- eixo: $\quad 6 \times 450+\frac{1.200 \times 0,26 \times 4,50}{2}=\frac{\operatorname{Cr} \$ 3.572,00}{\operatorname{Cr} \$ 12.301,00}$

Esta solução é $\operatorname{Cr} \$ 12.301-11.948=\operatorname{Cr} \$ 353,00$

- Cr\$353,00 mais caro que a obtida pela fórmula deduzida.

\section{CONCLUSÃO E RESUMO}

Foi apresentado o método de cálculo que permite achar uma solução eventual às críticas I e II de Burbidge.

A fórmula deduzida é de fácil aplicação para cálculos múltiplos com máquina de calcular comum, não necessitando de computador e permite a uma indústria de montagem em série, comprar dentro do menor custo fora do lote econômico. $O$ cálculo é realizado em função do item considerado básico, escolhido previamente.

E importante frisar em conclusão que o método não pretende aumentar custos mas compensar pela diminuição da obsolescência e do pessoal necessário para compra ou preparação o 
custo escondido do aumento do custo total anual de compra.

O custo de comprar "e o custo de ter estoque" são praticamente idênticos - o que de fato acontece pois na decisão de comprar por um lote econômico composto a soma será mínima quando os dois termos são iguais.

De fato:

$$
\frac{A_{i}}{Q_{i}}=N_{i} \begin{aligned}
& \text { e para todos os itens } N \text { é o mesmo, } \\
& \text { pela definição de compra em lotes } \\
& \text { múltiplos, }
\end{aligned}
$$

pois: $\quad f_{i} A_{1}=A_{i}$

\section{e}

$$
f_{j} Q_{1}=Q_{i}
$$

então o "custo de compra" será:

$$
\sum_{1}^{i} \frac{A_{i}}{Q_{i}} \times S=i \frac{A_{1}}{Q_{1}} \cdot S
$$

o custo de ter estoque será:

$$
\sum_{1}^{i} \frac{Q_{i} C_{i} \mid}{2}=\frac{Q_{1} l}{2} \sum_{1}^{i} f_{i} C_{i}
$$

pois $Q_{i}=f_{i} Q_{1}$

donde $\sum_{1}^{i}$ C.T.A.j $=i \frac{A_{1}}{Q_{1}} S+\frac{Q_{1} l}{2} \sum_{1}^{i} f_{i} C_{i}$

agora $i \frac{A_{1}}{Q_{1}} S=\frac{Q_{1} /}{2} \sum_{1}^{i} f_{i} C_{i}$

pois $Q_{1}^{2}=\frac{2 S . i . A_{i}}{/ \sum_{1}^{i} f_{i} C_{i}}=Q_{1}^{2}$ (formula do lote)

$\frac{i A_{1} S \sqrt{\frac{2 S}{1}} \sqrt{\frac{i A_{i}}{\sum_{i}^{i} f_{i} c_{i}}}, \sum_{1}^{i} f_{i} c_{i}}{\sqrt{\frac{2 S}{I}} \cdot \sqrt{\frac{i A_{1}}{\sum_{i}^{i} f_{i} C_{i}}}}=\frac{\sqrt{2}}{\sqrt{\frac{1}{1}}}$

então:

$$
\frac{\sqrt{i A_{1}} \sqrt{\sum_{1} f_{i} C_{i}} \sqrt{S} \sqrt{l}}{\sqrt{2}}=\frac{\sqrt{i A} \sqrt{\sum_{1} f_{i} C_{i}} \sqrt{S} \sqrt{i}}{\sqrt{2}}
$$

que demonstra a igualdade:

$$
\sum_{1}^{i} C . T . A_{. j}=2 \times i \frac{A_{1}}{Q_{1}} S
$$

Aplicando ao exemplo em questão:

$\sum_{1}^{i} C . T \cdot A_{\cdot j}=2 \times 3 \frac{3.600}{814} \times 450=$

$=\frac{2.700 \times 3.600}{814}=\frac{9.720 .000}{814}=11.941,00$

Este valor é praticamente idêntico ao conseguido anteriormente.

1 Burbidge, John $L$. The principles of production control. London, McDonald \& Evens Ltd. 1962.

2 Burbidge na p. 149 dá quatro razões por que ele não aceita o lote econômico de compras e de produção:

I. O inventário em estoques é determ inado como resultado aleatório de um grande número de cálculos independentes para diferentes componentes.

II. O LEC exige o uso de pedido em múltiplas fases aceitando-se altos custos de obsolescência e de administração.

III. O LEC eterniza a ineficiência do sistema de pedido.

IV. O LEC está em desacordo com a exigência da alta rotatividade dos estoques.

3 Caso Metalúrgica Farma Ltda. EAESP/FGV, P-619. Para meIhor esclarecer aos leitores este aspecto segue-se um pequeno trecho e um exemplo do caso em ep/grafe: "Usando o método de calcular S, pelo custo direto mais o custo da produção cessante, ficou demonstrado um aumento incrivel do custo de preparação, provocando um crescimento considerável do lote de produção. Henrique procurava então alguma regra lógica pela qual poderia trabalhar para não so não ter um estoque ficticiamente grande, por um valor alto de $S$, nem perdas de produção por um valor baixo. Exemplo: Assim, um produto fabricado na quantidade de 50 unidades/hora tinha vendas anuais de 16.200 unidades, para 0 jue havia necessidade de trabalhar 324 horas, ou seja, aproximadamente $\mathbf{4 0}$ dias, isto $e$, oito semanas. $O$ tempo de preparação era de 5 horas e custava, entre planejamento, mão-de-obra direta e tempo máquina, além de tempo de homens como ferramenteiro, $\mathrm{Cr} \$ 900,00$. O valor de custo do produto, que entrava em estoque, era de $\mathrm{Cr} \$ 256,00$. A contribuição do produto, por unidade, era de $\operatorname{Cr} \$ 250,00$. O custo do dinheiro, obsolescência, etc. era estimado em $25 \%$.

Calculando o lote econômico de produção pela maneira comum, dava:

$$
\begin{aligned}
Q=(16.200 \times 2 \times 900 / 0,25 \times 256)^{1 / 2} & =\frac{180 \times 30}{0,5 \times 16}= \\
& =5.400 / 8=675 \text { unidades. }
\end{aligned}
$$

Portanto, produzem-se $16.200 / 675=24$ vezes aproximadamente.

Fazendo o mesmo cálculo com a perda de contribuição, que corresponde a 5 horas vezes 50 unidades vezes $\operatorname{Cr} \$ 250,00=$ Cr\$62.500,00 temos:

$Q^{\prime}=(16.200 \times 2 \times 62.500,00 / 0,25 \times 256)^{1 / 2}=180 \times 250 / 0,5 \times$ $16=45.000 / 8=5.625$

Assim, produzir-se-iam, aproximadamente, três vezes por ano. 
Deixa-se de considerar alem dos $\operatorname{Cr} \$ 62.500,00$ os $\operatorname{Cr} \$ 900,00$ do custo direto, por serem insignificantes".

4 Considera-se conhecida a formula do lote económico de produção e compras; está alêm dos limites deste comentário discutir a formula ou outras delas derivadas.

5 Os métodos empregados são individuais, por exemplo, o Prof. Claude Machline recomendaria comprar no caso presente rodas e eixos três vezes por ano e caçambas seis vezes, mantendo uma espécie de multiplo de submúltiplo.

\section{BIBLIOGRAFIA}

Araújo, Jorge Siqueira de. Administração de materiais. 2. ed. São Paulo, Atlas, 1971.

Messias, Sérgio Bolsonaro. Manual de administração de materiais. Atlas.
Araújo, Jorge Siqueira de. Saiba comprar para a sua empresa. 2. ed. Atlas.

Heinritz \& Farrel. Compras. Atlas, 1972.

England, Wilbue B. O método de compras. Brasiliense, 1970.

Stansburg Stockton, R. Sistemas básicos de controle de estoques. São Paulo, Atlas, 1972.

Maynard, H. B. Manual de engenharia de produção. Ed. Edgard Blucher Ltda. 1970, capítulo de Neuschel sobre controle de estoques.

Machline, Sá Motta, Schoeps \& Weil. Manual de administração da produção. Rio de Janeiro, Fundação Getulio Vargas, 1969, v. 1. Capítulos sobre gestão de estoques e compras.

Zacarelli, Sérgio B.Programação e controle da produção. CEPAL, Livraria Pioneira Editora, 1967.

O CORREIO DA UNESCO CONJUNTURA ECONÔMICA (CE)

REVISTA DE ADMINISTRAÇÃO PÚBLICA (RAP) REVISTA DE DIREITO ADMINISTRATIVO (RDA) REVISTA DE CIÊNCIA POLÍTICA (RCP) REVISTA DE ADMINISTRAÇÃO DE EMPRESAS (RAE) ARQUIVOS BRASILEIROS DE PSICOLOGIA APLICADA (ABPA) CURRICULUM (CUR) REVISTA BRASILEIRA DE ECONOMIA (RBE) 